Christine Bracquenier

MoDyCo, UMR 7114

Université Charles-de-Gaulle - Lille 3

CNRS - Université Paris Ouest Nanterre La Défense

christine.bracquenier@univ-lille3.fr

\title{
Circonstants et spécifiants : vers une nouvelle analyse sémantico-syntaxique de la phrase russe
}

\section{Version de travail}

Les grammaires se contentent en général de proposer des listes de «compléments circonstanciels ", mais la difficulté n'est pas tant de lister et d'organiser ces catégories que de définir ce terme de la phrase. Le métaterme français fait intervenir la notion de complément, ce qui le rattache au verbe. Le russe ayant la faculté de construire des phrases sans verbe, je partirai de la notion de nexus pour construire une nouvelle typologie de la phrase russe en prenant en considération les notions de rection et de réquisition. La mise en ouvre de ces notions me permettra de formuler une définition du circonstant et de produire une nouvelle partition entre actants, spécifiants et circonstants.

\section{Problèmes de définition}

\subsection{La grammaire française}

Le «circonstanciel » de l'abbé Gabriel Girard ${ }^{1}$, devenu un «complément circonstanciel » pour Nicolas Beauzée ${ }^{2}$, est généralement défini au niveau de la sémantique, alors même que son appellation traditionnelle fait également référence à la syntaxe, et la liste des « compléments circonstanciels » fait percevoir le grand flou qui entoure cette notion ${ }^{3}$. Comme l'adverbe auquel il est étroitement associé ${ }^{4}$, le complément circonstanciel a aussi toujours été une catégorie résiduelle où l'on place tout ce que l'on ne réussit pas à ranger de manière sûre ailleurs, et les définitions négatives sont les plus fréquentes pour le circonstant ${ }^{5}$. G. Lazard produit une liste de critères, très réduite, qui caractérisent le circonstant, ou plutôt ne le caractérisent pas : le circonstant n'est ni requis, ni régi ; il n'entre pas en corrélation avec un indice actanciel du moins en russe (en français, les indices y et en sont tantôt actanciels, tantôt circonstanciels) ; les relateurs qu'il met en œuvre (prépositions et / ou marques casuelles) sont

\footnotetext{
${ }^{1}$ C'est l'abbé Gabriel Girard, d'ailleurs premier russisant francophone, qui est l'inventeur du terme «circonstanciel » en 1747: «Ce qu'on emploie à exposer la manière, le temps, le lieu, \& les diverses circonstances dont on assaisonne l'attribution gardera le nom de CIRCONSTANCIEL, puisque toutes ces choses y paroissent d'un air de Circonstance » (1747, Les Vrais principes de la langue française : ou La parole réduite en méthode, conformément aux loix de l'usage : en seize discours, tomes I et II, Paris, Le Breton. I : 60).

${ }^{2}$ N. Beauzée (1767, Grammaire générale, Paris, J. Barbou, t.2, chap. II, livre 3, p. 58) définit le complément circonstanciel par «le vers technique dont se servent les rhéteurs pour caractériser les diverses circonstances d'un fait : quis, quid, ubi, quibus auxiliis, cur, quomodo, quando. Le premier mot quis est le seul qui ne marque aucun complément, parce qu'il indique au contraire le sujet; mais tous les autres indiquent autant de compléments différents ».

${ }^{3}$ Cf. par exemple la définirion donnée par Grevisse (1969: 148) : «Le complément circonstanciel précise l'idée $\mathrm{du}$ verbe en marquant la connexion de l'action avec un repère (temps, lieu, etc.) situé autour d'elle dans le monde des phénomènes ». L'italique est de Grevisse. Grevisse (1969 : 148-149) en donne 32 sortes. Dans la dernière édition (la $14^{\mathrm{e}}$, refondue par A. Goosse en 2008), la liste est limitée à huit distinctions sémantiques (p. 390)

${ }^{4} \mathrm{~A}$ tort, à mon avis. De nombreux adverbes n'ont rien à voir avec l'expression de circonctances.

${ }^{5}$ Par exemple, Guimier (1993:15) : «Un circonstant est un constituant satellite du verbe qui ne remplit aucune des fonctions sujet, attribut, complément essentiel, direct ou indirect ».
} 
des plus divers ; sa place n'est pas fixée grammaticalement ${ }^{6}$. Le circonstant, qui pour certains (notamment, Tesnière 1976, Guimier 1993, Grevisse \& Goosse 1993, 2008 ${ }^{7}$ ) reste attaché au verbe, est toujours considéré comme un élément facultatif, accessoire, secondaire de la phrase et l'on avance pour preuve le fait que l'on peut aisément le déplacer ou le supprimer. Ceci est à peu près acceptable si l'on parle de la phrase. Mais dès que l'on passe au niveau de l'énoncé ${ }^{8}$, on s'aperçoit que les possibilités de déplacement ou de suppression innocentes du circonstant sont extrêmement réduites ${ }^{9}$. La distinction entre complément « essentiel »vs «non essentiel », mise en œuvre dans les années 1990, éloigne peu à peu le complément circonstanciel du verbe et il devient un « complément de phrase ${ }^{10}$. Selon moi, la question de savoir si le circonstant est un complément de verbe ou de phrase n'est pas pertinente parce que j'estime que le circonstant n'est pas un « complément ».

\subsection{La grammaire russe}

Depuis M. V. Lomonosov qui a distingué des parties du discours principales et accessoires ou auxiliaires (главные vs вспомогательные или служебные части человеческого слова) ${ }^{11}$, les grammaires russes divisent les termes de la proposition en termes principaux (главные члень предложения) et secondaires (второстепенные члень предложения) qui « servent à expliciter les termes principaux de la proposition ${ }^{12}$ et que la Grammaire de l'Académie de 1980 préfère nommer «expansions » (расnространяюшие члень предложения). Il existe trois types de termes secondaires, les termes principaux étant le sujet (подлежащеe) et le prédicat (сказуемое): le complément (дополнение), le déterminant (определение) et la circonstance (обстоятельство) qui est définie comme l'élément «qui se rapporte à un terme de la proposition exprimé par un verbe, un substantif verbal, un adjectif ou un adverbe et qui sert à caractériser l'action ou l'attribution par rapport à sa qualité ou son intensité, à indiquer la manière dont se réalise l'action, le temps, le lieu, la cause, le but, la condition avec lesquels sont liées l'action ou la manifestation de l'attribution $»^{13}$.

La Grammaire de l'Académie de 1980 évoque la difficulté de déterminer avec précision à quelle catégorie de termes secondaires appartient tel ou tel constituant de la proposition :

Стреляют из окна: из чего? - дополнение и откуда? - обстоятельство;

Поговорим перед ужином: перед чем? - дополнение и когда? - обстоятельство ${ }^{14}$.

Les auteurs de manuels scolaires sont unanimes sur le procédé : il faut poser des questions, et cela apparaît dans la définition même des « circonstances » :

\footnotetext{
${ }^{6}$ Lazard $1994: 81$ sqq.

${ }^{7}$ A. Goosse (Grevisse \& Goosse 2008 : 392) réfute l'idée de «complément de phrase » pour les compléments non essentiels, arguant du fait que « le complément de phrase fait partie de la phrase, alors que le complément du verbe ne fait pas partie du verbe », et il donne aussi des exemples où les compléments adverbiaux non essentiels « accompagnent » des verbes prédicats de propositions (et pas seulement de phrase), des infinitifs, des gérondifs, des participes, des adjectifs, des noms.

${ }^{8}$ J'appelle « énoncé » une phrase produite en situation d'énonciation.

${ }^{9}$ J'ai montré en effet que le circonstant joue un rôle important dans la cohérence du texte (Bracquenier 2009) et qu'il est des cas où on ne peut pas le supprimer sans que la phrase devienne agrammaticale (Bracquenier 2011).

${ }^{10}$ Cf. par exemple Guiraud 1961, La Grammaire, Paris, P.U.F., «Que sais-je ? » n 788 ; Wagner et Pinchon 1962 \& 1991, Grammaire du Français classique et moderne, Paris, Hachette ; Riegel et al., 1994, Grammaire méthodique du français, Paris, P.U.F., « Quadrige ».

${ }^{11}$ Lomonosov 1755, § 45-47. L'adverbe («pour une représentation concise des circonstances ») et la préposition («pour montrer l'appartenance des circonstances aux choses et aux actions ») (§ 46) relèvent des parties du discours accessoires.

${ }^{12}$ Grammatika russkogo jazyka 1960, II, 1 : 520.

${ }^{13}$ Grammatika russkogo jazyka 1960, II,1: 521.

${ }^{14}$ Russkaja grammatika 1980, II : 91. Mes informateurs n'acceptent pas la question Из чего стреляют? si la réponse attendue est из окна; la réponse à la question serait из ружья. Quant à Перед чем поговорим?, il ne peut guère s'agir que d'une reprise, si l'interlocuteur a mal compris, par exemple.
} 
Обстоятельств а - это второстепенные члены, которые указывают на место (отвечают на вопросы где? куда? откуда?), время (когда?), образ (способ) действия (как?), например: 1) Виднелись горы вдалеке. (Н.) 2) Mы спускалсиь в долину. (П.) 3) Сумерки быстро сгущзались. (Арс.)

Обстоятельства могут иметь и другие значения, например: Он пропустил занятия (п о ч е м у? ) по болезни - причина.

Обстоятельства чаще всего выражаются наречиями или существительными в разных падежах (кроме именительного) и обычно относятся к сказуемому ${ }^{15}$.

Ainsi, si le terme de la proposition répond à une question posée à l'aide d'un pronom interrogatif à un cas oblique, accompagné ou non d'une préposition, il est un complément. S'il répond aux questions formulées au moyen d'un adverbe, il est une circonstance. Pourtant, certains syntagmes se prêtent aux deux types de questionnement, c'est ce que montre la Grammaire de l'Académie de 1980, et certains syntagmes reconnus comme des circonstances répondent difficilement à la question posée par un adverbe :

В словосочетаниях сидел в комнате, побледнел от страха, летел птицей сушествительные в косвенных падежах (с предлогом и без предлога) в комнате, от страха, птицей следует признать обстоятельствами, а не дополнениями. Существительное (с предлогом) в комнате обозначает место (сидел - где ? - в комнате), существительное (с предлогом) om страха обозначает причину (побледнел - почему? - от страха), существительное птицей обозначает способ (образ) действия (лететь - как ? - птицей). К дополнениям же относим только слова со значением предметности и отвечающие на вопросы косвенных падежей ${ }^{16}$.

Pour le deuxième énoncé, ne peut-on pas plutôt interroger à l'aide d'une question du type « cas oblique »? Il est peu probable [...]

La notion de complément essentiel avait déjà été évoquée dans la Grammaire de l'Académie de 1960 :

$[\ldots]^{17}$.

Si l'on considère les exemples вбить гвоздь в стену, налить молоко в стакан, вынуть тетрадь из портфеля, ces « ajouts nécessaires » sont exactement ce que D.È. Rozental' \& M.A. Telenkova appellent des «compléments circonstanciels» (обстоятельственные дополнения $)^{18}$.

La Grammaire de l'Académie de $1960[\ldots]^{19}$.

Dans ce petit paragraphe et même si le terme de circonstance n'y est pas employé, les auteurs opèrent une distinction entre la «circonstance de verbe » et la «circonstance de proposition ». C'est cette distinction de portée qui permet d'apprécier la différence de fonction entre différents syntagmes prépositionnels ; c'est elle qui éloigne la circonstance du verbe et en fait une fonction à analyser non plus au niveau du prédicat, mais au niveau de la phrase.

\section{Proposition de définition du circonstant}

Ainsi, à partir du dernier tiers du $\mathrm{XX}^{\mathrm{e}}$ siècle, et de manière plus appuyée pendant sa dernière décennie, l'idée selon laquelle certains compléments que la grammaire traditionnelle rangeait parmi les circonstanciels n'en étaient peut-être pas s'est développée et affinée. Il convient alors de diviser les «ex-compléments circonstanciels» en au moins deux grandes catégories : ceux qui restent des «compléments circonstanciels », accessoires, facultatifs, etc.,

\footnotetext{
${ }^{15}$ S.E. Krjučkov \& L.Ju. Maksimov, Russkij jazyk dlja 5-8 klassov večernej (smennoj) školy, 1976 :18.

${ }^{16}$ A.S. Matijčenko, Grammatika russkogo jazyka, Čast' 2-aja : sintaksis, 1955 : 43.

17 Grammatika russkogo jazyka 1960, II, 1 : 118.

18 Rozental' \& Telenkova (1972 : 208) : «Второстепенный член предложения, в котором совмещаются значения объектное и обстоятельственное (чаще всего пространственное). Хранить документы 6 портфеле ( в чем? где?) ». Je regrette qu'ils ne soient pas allés plus avant dans leur analyse et qu'ils n'aient pas constaté le caractère obligatoire (essentiel) de ce type de complémentation.

${ }^{19}$ Grammatika russkogo jazyka 1960, II,1 : 522-523.
} 
et ceux qui ne peuvent plus prétendre à cette appellation parce qu'ils sont des compléments du verbe obligatoires, essentiels, requis et / ou régis.

Dans la mesure où le statut d'un complément est déterminé en fonction du degré de son attachement au verbe, il m'a semblé qu'il fallait s'intéresser avant tout au verbe lui-même. La langue russe a cependant la capacité de construire des propositions autour d'une partie du discours autre que le verbe. Pour cette raison, je substitue au «verbe »le terme de nexus ${ }^{20}$ défini par J.-P. Sémon comme l'« organisateur morpho- et sémiosyntaxique de la phrase ». Cette notion permet d'édifier une nouvelle typologie des phrases russes, fondée sur le nexus qui prédétermine au niveau sémantique et / ou syntaxique la présence possible ou obligatoire d'un certain nombre d'éléments qui dépendent de lui ${ }^{21}$ et que j'appellerai ses « dépendants ». Ces éléments sont des actants régis et requis ou non requis, des adjets ${ }^{22}$ requis mais non régis, et des spécifiants ${ }^{23}$, pour lesquels les notions de rection et de réquisition ne sont pas pertinentes, mais qui sont, en tout état de cause, sélectionnés par le nexus. Le nexus et ses dépendants forment le noyau de l'énoncé. Le circonstant, comme son nom l'indique, est autour du noyau. Mais il faut bien se garder d'interpréter "autour» comme un espace extérieur, périphérique et de là accessoire, comme cela a été fait jusqu'à présent. «Autour » désigne ici un espace qui entoure et englobe le noyau. Je propose la définition suivante du circonstant :

Le circonstant est l'expression sémantique et morpho-syntaxique du cadre spatial, temporel ou causal ${ }^{24}$ dans lequel le noyau de l'énoncé est valide. Il n'est sélectionné ni sémantiquement ni syntaxiquement par le nexus.

Le circonstant n'est en aucun cas un « complément », ni du verbe, ni de la proposition. C'est lui qui pose, qui circonscrit le cadre à l'intérieur duquel l'énoncé est vrai. En ce sens, le nexus est directement impliqué, mais non pas parce qu'il sélectionnerait une quelconque circonstance. C'est, à mon avis, l'inverse : c'est le circonstant qui « sélectionne », d'une certaine manière, le nexus et ses dépendants.

\section{Les éléments de la structure phrastique}

Les éléments de la phrase sont le circonstant, le nexus et ses dépendants (actants, adjets et spécifiants). La prise en compte de la nature du nexus et de ses exigences au niveau des dépendants permet d'établir une typologie sémantique et morpho-syntaxique de la phrase russe qui ne se fonde pas sur la bipartition en phrases monorèmes/dirèmes ${ }^{25}$.

\subsection{Le nexus}

Le nexus peut être un verbe (autre que la copule) à une forme personnelle définie, s'accorde avec l'actant sujet au nominatif, mais ne le réquisitionne pas, ou à une forme finie impersonnelle, sans actant lorsque le verbe exprime un phénomène météorologique (морозит, светает) ou avec un actant (exprimé ou non) dont le cas est régi par le nexus (маму знобит, пахнет рыбой, хватает хлеба, маме не спится). Les structures du type

\footnotetext{
${ }^{20}$ Sémon 2004 : 113.

${ }^{21}$ C'est la théorie de la valence de L. Tesnière, mais qui nécessite une révision.

${ }^{22}$ Le terme créé dans cette acception par J. Feuillet (1980 : 26) est repris par G. Lazard (1994 : 17).

${ }^{23}$ Ce terme est le mien.

${ }^{24}$ Le circonstant causal comprend la cause agréée (c'est-à-dire, la cause à proprement parler), la conséquence, le but, la condition, la concession.

${ }^{25}$ Cette bipartition des phrases russes est intéressante, mais elle repose sur l'idée, qui remonte aux théoriciens antiques, que toute phrase est construite sur le binôme onoma - rhêma (nom - verbe ; sujet - prédicat), ce qui n'est pas le cas du russe ; de ce fait, la linguistique russe a dû s'adapter et proposer des phrases qui ne présentent que rhêma. Cette typologie fondée sur la présence ou non d'un sujet syntaxique, range dans des catégories très différentes des phrases au fonctionnement similaire (par exemple, Я читаю est une phrase dirème alors que Читаю est monorème).
} 
деревья свалило ураганом оù les deux actants, objet et instrument, sont régis et requis par la forme du nexus, entrent dans cette catégorie. Enfin, le verbe peut être à l'infinitif, la structure est impersonnelle, l'infinitif sélectionne un actant au datif qui peut ne pas être exprimé.

Lorsque le nexus est la copule, il s'agit de phrases attributives, avec un actant sujet au nominatif ; au présent la copule est absente de la phrase, qui prend l'apparence d'une phrase nominale, mais il me semble plus raisonnable de ne pas séparer dans la nomenclature les phrases qui sont au présent et celles qui sont au passé ou au futur, c'est-à-dire avec la copule physiquement présente et qui sont rangées dans les phrases verbales. J'inclus dans ce type de phrases celles qui comportent le présentatif $э m o^{26}$ et, tout en reconnaissant leurs spécificités syntaxiques, les phrases de possession (avec un actant exprimant le possesseur ou la localisation ${ }^{27}$ ), qu'elles soient affirmatives (avec un actant sujet) ou négatives (avec un actant objet), ainsi que les phrases de «non-être » : la copule sous sa forme négative s'associe à un pronom ou un adverbe pour former un nexus négatif dont dépendent un actant au datif et un actant qui est un verbe à l'infinitif (мне нечего делать).

Le nexus peut aussi être un substantif. Il s'agit des phrases narratives d'exposition :

Зима. Вечер. Ветер. Пыль. (А. Слаповский, Пыльная зима)

mais aussi des phrases d'ostension :

- А вот и наша Лихаревка, - закричал Скворцов. (И. Грекова, На испытаниях)

ou encore d'apostrophe (Ваня!); c'est le cas aussi des enseignes (Овощи). Toutes ces phrases remplissent une fonction déictique et toutes (sauf les phrases d'exposition) ne sont viables qu'au présent. Elles sont donc exclusivement nominales. Mais on peut légitimement se poser la question de savoir si les phrases d'exposition mises au passé ou, éventuellement, au futur, où la copule s'accorde avec le substantif au nominatif, sont des phrases verbales au même titre que celles qui sont examinées ci-dessus ou si elles restent des phrases nominales avec une marque temporelle. La structure sémio-syntaxique des phrases d'exposition et des phrases attributives ou possessives est différente : la modalité interrogative et la variation temporelle, si elles sont possibles en théorie, sont très rares, et, comme toutes les autres phrases dont le nexus est un substantif, elles ne peuvent être soumises à la modalité négative ; ces caractéristiques me conduisent à considérer les phrases d'exposition, au présent ou à tout autre temps, comme des phrases dont le nexus est le substantif.

Le nexus est un numéral et les phrases expriment l'âge ou la quantité :

- Слуг у меня теперь нет. Было двое парней, да остались там, на снегу лежать, - горестно вздохнул Вальзер. (Б. Акунин, Алтын-Толобас)

Enfin, le nexus est un adverbe déadjectival (жарко), un adverbe ou une locution adverbiale parfois appelés «prédicatifs autonomes» (пора, не до смеху) ou encore un adverbe modal, comme надо, нужно, etc.

\subsection{Les actants}

Les actants présentent deux caractéristiques : la rection (forme imposée) et la réquisition (présence imposée), qui dépendent de la nature et de la forme du nexus ${ }^{28}$. On obtient potentiellement quatre cas de figure.

L'actant régi et requis. C'est le cas de «compléments » de nombreux verbes, en français et en russe, qui exigent la présence d'une complémentation et lui imposent une

\footnotetext{
${ }^{26}$ Comme les phrases attributives, elles acceptent les modalités négative et interrogative.

${ }^{27}$ L'actant qui exprime le possesseur, dans ce type de phrase, prend la forme d'un syntagme de localisation (comparez y меня есть рояль, у меня в комнате есть рояль еt в театре есть рояль).

${ }^{28}$ Les ouvrages qui traitent de la rection des verbes russes ne manquent pas ; cependant, ils ne concernent que les verbes (les adverbes, par exemple, ne sont généralement pas pris en compte) et surtout il n'est jamais indiqué si la complémentation est obligatoire ou non ; or, pour les apprenants étrangers, il est important de connaître cette caractéristique du nexus, d'autant plus qu'il n'y a pas forcément d'équivalence syntaxique dans ce domaine d'une langue à l'autre. C'est un travail que je compte mener dans les années à venir.
} 
forme ; par exemple, les verbes rencontrer / встречать ne peuvent être employés seuls : *elle rencontre / *она встречаem, et la forme imposée est un objet, direct en français, à l'accusatif en russe : elle rencontre un аті Іона встречает друга; toute autre forme est impossible. Lorsque le nexus est un numéral il fonctionne avec un ou deux actants régis et la présence de l'un des deux au moins est obligatoire.

L'actant régi et non requis. C'est le cas du sujet en russe. Comme le montre la typologie des phrases présentée ci-dessus, nombre d'entre elles fonctionnent sans qu'un actant sujet soit requis. Néanmoins, dans les phrases dont le nexus est un verbe à une forme personnelle ou la copule, on peut dire que le sujet est régi. Le sujet laisse une place actancielle vide lorsqu'il n'est pas contextuellement défini, c'est alors l'indice actanciel du verbe qui dénote le caractère inclusif ou exclusif des participants humains. Lorsque le nexus est un verbe impersonnel (à l'exception des phénomènes météorologiques qui ne demandent aucun actant) ou à une forme impersonnelle, ou s'il est un adverbe, la présence d'un actant n'est pas requise, mais sa forme est imposée par la forme et/ou la nature du nexus.

L'actant non régi mais requis. Il s'agit de ce que J. Feuillet et G. Lazard nomment l'adjet. La présence d'un actant est obligatoire mais sa forme n'est pas imposée par le nexus : $J$ 'habite en France, à Lille, dans un appartement, avec ma fille, chez mes parents, etc. On ne peut pas parler d'adjet avec le verbe жumb, parce qu'il peut s'employer seul, mais le verbe проживать exige un actant, soit un objet, soit un actant de localisation, et les deux sont possibles simultanément.

L'actant non régi et non requis. G. Lazard explique que ce sont les caractéristiques du circonstant, et on sort donc du cadre de l'actance proprement dite. Il me semble pourtant que certains éléments de la phrase, appelés traditionnellement «compléments » et rangés dans la catégorie des «compléments circonstanciels » pourraient être analysés autrement. J'appelle ces termes de la phrase des spéficiants.

\subsection{Les spécifiants}

Ils dépendent aussi du nexus et peuvent être requis et régis ou non régis, et ni régis ni requis. Les spécifiants requis sont considérés dans les grammaires traditionnelles, aussi bien russes que françaises, tantôt comme des compléments circonstanciels, tantôt comme des compléments d'objet; le second type est toujours classé parmi les compléments circonstanciels. Ce qui m'amène à proposer cette notion, c'est que le spécifiant actualise le procès de manière interne et les sèmes du spécifiant sont déjà entièrement compris dans la sémantique du nexus; ou bien il indique la manière dont se déroule le procès - durée, itération, manière, moyen, instrument, comitativité.

\subsubsection{Les spécifiants requis et régis ou non régis.}

Этот шкаф весит 100 килограмм. *Этот шкаф весит тяжело

Он бежал / пробежал 100 метров. *Он бежал далеко. ?Он пробежал далеко.

mais :

Это пальто стоит 200 евро. Это пальто стоит дорого.

et les variantes syntaxiques sont nombreuses avec le verbe длиться $^{29}$ : длиться 500 дней, всю зиму, до зари, без конца, часами, долго, Ø :

Вместо запальчивости и гнева во мне продолжала длиться смиренная тишина. (В. Маканин, Андеграунд, или герой намего времени)

Cet exemple montre que le spécifiant peut ne pas être exprimé, mais d'une part, l'absence de spécifiant est «compensée » par l'indication d'une phase; d'autre part son absence est porteuse de sens et elle contraint l'énonciataire à rétablir uniquement un seul type de valeur :

\footnotetext{
${ }^{29}$ En français aussi, le verbe durer permet plus de variantes syntaxiques pour son spécifiant que les autres nexus de ce type sémantique : Les travaux dureront tout l'hiver/trois mois/longtemps/Ø.
} 


\subsubsection{Les spécifiants ni requis ni régis}

Il s'agit de ce que j'appelle les spécifiants aspectuels (durée, itération), de localisation spatiale ou temporelle, de manière, d'instrument/moyen et de comitativité. Ces spécifiants ni requis ni régis sont néanmoins sélectionnés sémantiquement par le nexus.

On a occupé l'usine pendant trois jours / On a occupé l'usine en trois jours ${ }^{30}$. [...]

Les spécifiants de localisation spatiale ou temporelle interviennent avec des verbes de déplacement ou d'occurrence (случиться, иметь место, etc.). et ne font que nommer un lieu/un moment choisi à l'intérieur d'un paradigme présélectionné par le nexus.

La question de savoir si le «complément de manière » est un complément circonstanciel a fait couler beaucoup d'encre ${ }^{31}$, or de nombreux linguistes s'accordent à considérer que «l'adverbe [de manière] est au verbe ce que l'adjectif est au nom ${ }^{32}$ : au même titre que l'adjectif peut déterminer ou qualifier un substantif, l'adverbe/le syntagme de manière ne fait que déterminer, qualifier le nexus seul ou le nexus et ses dépendants. Une réflexion semblable peut être menée à propos des «compléments » d'instrument ou de moyen (observons les verbes de déplacement qui lexicalisent le moyen de transport (examb est aller-avec-unmoyen-de-locomotion-sur-terre) ou des verbes comme застрелить $(=$ убить из огнестрельного оружия) qui montrent que les sèmes des spécifiants sont bien compris dans le nexus et qu'il ne peut s'agir d'un circonstant).

Quant au «complément d'accompagnement », il ne peut que «participer » au procès et à ce titre il ne peut être un circonstant. Il est venu avec sa femme ${ }^{33}$ implique que sa femme et lui soient venus ensemble, il n'y a donc qu'un seul procès; le «complément d'accompagnement » équivaut à une coordination des sujets : Sa femme et lui sont venus; en russe la tournure comitative est préférée à la coordination et non seulement elle génère un accord pluriel du nexus, mais elle intègre la pluralité des participants dans le sujet :

На другой день они с дядей должны были уехать далеко на юг [...] (Б. Пастернак, Доктор Живаго)

< Le lendemain son oncle et lui devaient partir loin dans le sud [...] >

\subsection{Les circonstants}

Des tests sémantico-syntaxiques ayant pour finalité de distinguer les circonstants des « compléments essentiels » sont proposés dans les ouvrages français traitant des circonstants. Certains tests ne sont pas pertinents pour le russe, comme par exemple le détachement (par une virgule), ou le clivage qui n'est guère utilisé en russe si ce n'est pour l'expression de la cause (externe au sujet humain du nexus). Le test de la passivation, qui devrait permettre de montrer la différence de comportement entre un complément d'objet et un autre terme de la phrase au même cas ${ }^{34}$, fonctionne difficilement en français et n'est pas réalisable la plupart du temps en russe, ne serait-ce que parce que la formation du passif est limitée et parce que l'accusatif sans préposition ne peut desservir l'expression d'un circonstant tel que défini plus haut $^{35}$. Le test de la pronominalisation n'est pas probant en russe ${ }^{36}:$ le détachement de l'objet ou du circonstant ne correspondent pas à la norme syntaxique. On peut parler de pronominalisation également lorsqu'il s'agit d'anaphore, mais tout actant, spécifiant, ou circonstant peut être repris par un anaphorique, le test n'est donc pas pertinent. Les deux tests

\footnotetext{
${ }^{30}$ Ces deux exemples sont donnés par Mélis $1983: 27$.

${ }^{31}$ Cf. H. Yvon, 1960, «Un peu d'histoire à propos du complément de circonstance », Le français moderne, t.28, Paris, D'Artray, p. 1-5.

${ }^{32}$ Tesnière 1945 : 147 ; P. Garde (Grammaire russe, Phonologie et morphologie, Paris, IES, 1998, p. 291) parle, pour les adverbes en /-o/ et /-i/ du russe, d' « épithète[s] du verbe ».

${ }^{33}$ Exemple cité par Nøjgaard $1993: 325$.

${ }^{34}$ Il lit le journal le matin $=>$ Le journal est lu par lui le matin/*Le matin est lu par lui le journal.

${ }^{35}$ Он читает газету => Газета прочитана им. / Он читает все утро => *Bсе утро прочитано им.

${ }^{36}$ En français : Le journal, il le lit le matin. / Le matin, il lit le journal. => * Le matin, il le lit le journal.
} 
qui sont mis en avant pour caractériser le circonstant sont l'effacement et la mobilité. Certes, on peut supprimer un circonstant sans que cela porte grand préjudice à l'intégrité de la phrase, mais aussi certains objets, aussi bien en russe qu'en français. Quant à la mobilité, ce critère n'est pas pertinent pour le russe : tous les termes de la phrase sont mobiles ${ }^{37}$. Enfin, deux tests, peu utilisés, sont pourtant plus révélateurs. Le premier, «Qu'est-ce qui se passe ?» ${ }^{38}$, permet de distinguer les circonstants (qui répondent positivement au test) des actants et des adjets, et j'ajoute, des spécifiants qui ne peuvent se soumettre à une telle interrogation, et il met en évidence leur « indépendance » par rapport au nexus :

А еще через час неизвестный гражданин явился в квартиру номер одиннадцать [...]. (М. Булгаков, Мастер и Маргарита)

Что случилось через час? Неизвестный гражданин явился в квартиру номер одиннадцать.

*Что случилось в квартиру/ в квартире номер одиннадцать? Неизвестный гражданин явился.

Le dernier test est celui de la portée de la négation ${ }^{39}$. Lorsque le nexus nié est accompagné d'un actant ou d'un spécifiant, la négation porte sur l'ensemble $<$ nexus+actant/spécifiant $>$ et le spécifiant pourrait être supprimé, cela ne changerait ni le signe du nexus, ni la vérité de l'énoncé :

- Тш... барин. Сегодня к Вере Павловне не приезжайте ночью, потому ихний муж не едет в Москву. (А. Аверченко, Магнит)

Le mari ne part pas, ni à Moscou, ni ailleurs, la négation porte sur <едет (в Москву)>, la localisation exprimée fait corps avec le nexus. Avec un circonstant, la situation est bien différente : si le signe du circonstant est stable (s'il correspond à ce qui est effectivement énoncé), alors celui du nexus l'est aussi ; si le circonstant tombe sous l'emprise de la négation, alors le signe du nexus est l'inverse de celui qui est énoncé dans la phrase linéarisée :

К «Арсеналу» она относилась хорошо, ведь мы работали четко по плану и никогда никого не подводили. [...] И вот, придя к ней и выслушав поздравления с упешным участием в таком почетном мероприятии, как Олимпиада, я воспользовался моментом и посетовал на то, что наш ансамбль не работает в Москве.

La négation porte sur le circonstant. Son signe est inverse de celui qui est énoncé et cela produit l'inversion du signe du nexus. Le groupe travaille, mais pas à Moscou. La négation porte certes sur <работает в Москве> (le groupe ne fait pas «travailler à Moscou »), mais en aucun cas elle ne porte sur le nexus seul (le groupe travaille). Cela confirme deux de mes hypothèses : d'abord, c'est dans le cadre fixé par le circonstant que le nexus et ses dépendants sont valides : c'est à Moscou que « наш ансамбль не работает» est vrai. Ensuite, le circonstant n'est pas toujours facultatif; en effet, on ne peut pas supprimer ici le circonstant sous peine de modifier totalement la vérité de l'énoncé : наш ансамбль не работает в Москве $\neq$ наш ансамбль не работает.

\section{Conclusion}

La prise en compte des caractéristiques sémantiques et syntaxiques du nexus, qui est l'élément organisateur de l'énoncé, permet de distinguer les différents types d'actants et de spécifiants qui dépendent de lui et de présenter ainsi une typologie des phrases russes : le schéma est exposé ici de manière théorique, il doit être développé et affiné par une présentation détaillée qui indique pour chaque type sémantique et morpho-syntaxique de nexus quels acatants et spécifiants sont attendus. Des tests sémantico-syntaxiques mettent en évidence ce qui différencie un circonstant en particulier d'un spécifiant, mais aussi d'un actant. Le circonstant est défini comme l'expression du cadre spatio-temporel et causal dans lequel le nexus et ses dépendants sont valides n'est pas un complément.

\footnotetext{
${ }^{37}$ Je rappelle que suppression et déplacement ne sont pas innocents et la structure informative de l'énoncé et la cohérence d'un texte sont modifiées par la suppression ou le déplacement d'un terme.

${ }^{38}$ Ce test est proposé pour le français par Leeman 1998 : 47-48.

${ }^{39}$ Ce test est utilisé par Nøjgaard 1993 : 323, mais le choix de ses exemples n'est pas convaincant et fausse, selon moi, ses conclusions (cf. Meunier-Bracquenier 2005 : 490).
} 


\section{Bibliographie}

BRACQUENIER C., 2009, «Le rôle des circonstants dans la cohérence du discours en russe contemporain », in BreuILlard J., ThOMAS P.-L., WLODARCZYK H., éd., La cohérence du discours dans les langues slaves, Linguistique théorique et textuelle, Revue des Études slaves, tome LXXX, fascicule 1-2, Paris, Institut d'études slaves, p. 59-71.

ID., 2011, «Le circonstant est-il un terme facultatif de la phrase russe », Revue des Études slaves, Paris, Institut d'Études slaves, LXXXII, fascicule 2, p. 295-309.

ID., 2012, "Transitivité vs intransitivité : sémantique et syntaxe du syntagme à l'accusatif », in V. BELIAKOV \& C. BRACQUENIER (éd.), La linguistique russe : une approche syntaxique, sémantique et pragmatique, Slavica occitania $\mathrm{N}^{\circ} 34$, Toulouse, Université de Toulouse-Le Mirail, p. 133-156.

FEUILLET J., 1980, «Les fonctions sémantiques profondes » in Bulletin de la Société de Linguistique, tome 75, fasc. 1, Paris, Klincksieck, p. 1-37.

GRAMMATIKA RUSSKOGO AZYKA, v dvux tomax, 1960, [Grammaire de la langue russe], V. V. Vinogradov (red.), Moscou, AN SSSR

GREVISSE M., 1969, Le Bon Usage. Grammaire française avec des remarques sur la langue française d'aujourd'hui, Gembloux-Paris, Duculot-Hatier.

GREVISSE M., Goosse A., 2001 13 , 2008 ${ }_{14}$, Le Bon Usage. Grammaire française, Gembloux, De Boeck-Duculot.

GUIMIER Cl. et al., 1993, 1001 circonstants, Caen, Presses Universitaires de Caen.

LAZARD G., 1994, L'actance, Paris, Presses Universitaires de France.

LEEMAN D., 1998, Les circonstants en question(s), Paris, Editions Kimé.

Lomonosov M.V., 1755, Rossijskâ̂ grammatika [Grammaire russienne], Saint-Pétersbourg, Académie Impériale des Sciences.

MELIS L., 1983, Les circonstants et la phrase, Louvain, Presses universitaires de Louvain.

MEUNIER-BRACQUENIER C., 2005, Contribution à l'étude du circonstant en russe contemporain, Lille, ouvrage original d'HDR.

NACIONAL'NYJ KORPUS RUSSKOGO AZYKA, ruscorpora.ru

NØJGAARD M., 1993, Les adverbes français, essai de description fonctionnelle, tome 2, Copenhague, Munksgaard.

Rozental' D. Ė., TelenKova M. A., 1972, Spravočnik lingvističeskix terminov [Mémento des termes linguistiques], Moscou, Prosveščenie.

RUSSKAA GRAMMATIKA, v dvux tomax, 1980, [Grammaire russe], N. Ju. Švedova (red.), Moscou, AN SSSR.

SEMON J.-P. (2004), « Ordre des mots et syntaxe dépendantielle (l'adjectif russe) », in COTTE P., Dalmas M., WlodarczyK H. (éds), Énoncer. L'ordre informatif dans les langues, Paris, L'Harmattan, p. 111-131.

TESNIERE L., 1945 (1934), Petite grammaire russe, Paris, Didier.

ID., 19762 (1959), Éléments de syntaxe structurale, Paris, Klincksieck. 